PROCEEDINGS OF THE

AMERICAN MATHEMATICAL SOCIETY

Volume 133, Number 10, Pages 2927-2937

S 0002-9939(05)07908-6

Article electronically published on April 25, 2005

\title{
ON THE WARING-GOLDBACH PROBLEM FOR SEVENTH POWERS
}

\author{
ANGEL V. KUMCHEV
}

(Communicated by Wen-Ching Winnie Li)

\begin{abstract}
We use sieve theory and recent estimates for Weyl sums over almost primes to prove that every sufficiently large even integer is the sum of 46 seventh powers of prime numbers.
\end{abstract}

\section{INTRODUCTION}

Let $k$ be a natural number and let $H(k)$ denote the least integer $s$ such that the diophantine equation

$$
p_{1}^{k}+p_{2}^{k}+\cdots+p_{s}^{k}=n
$$

is soluble in primes $p_{1}, \ldots, p_{s}$ for all sufficiently large integers $n$ satisfying certain local conditions. The local conditions are designed to exclude degenerate cases, in which (1.1) reduces to a similar equation in fewer unknowns. For example, since every representation of an even integer $n$ as the sum of three primes reduces to a representation of $n-2$ as the sum of two primes, we study (1.1) with $k=1$ and $s=3$ only when $n$ is odd.

In 1937 I. M. Vinogradov [16 found a new method for estimating sums over primes, which he used to prove that $H(1) \leq 3$. Hua 4 used Vinogradov's method to establish the bound

$$
H(k) \leq 2^{k}+1 \quad(k \geq 1),
$$

which is still the best result known for $k \leq 3$. Later, work of Vinogradov, Hua, and Davenport from the 1940s and 1950s (see Hua [5]) and a technique in Waring's problem developed in the mid-1980s by Thanigasalam [11, 12] and Vaughan [14] led to a series of improvements on (1.2) for $k \geq 4$. In particular, it was known by the late 1980s that

$$
H(4) \leq 15, \quad H(5) \leq 23, \quad H(6) \leq 33, \quad H(7) \leq 47, \quad H(8) \leq 63, \quad H(9) \leq 83 .
$$

Recently, Kawada and Wooley [] showed that

$$
H(4) \leq 14 \text { and } H(5) \leq 21 .
$$

The main innovation in $[7$ is the use of minor arc estimates stemming directly from sharp estimates for exponential sums over primes rather than from estimates for artificially introduced exponential sums over consecutive integers. The purpose of

Received by the editors May 17, 2004 and, in revised form, June 10, 2004.

2000 Mathematics Subject Classification. Primary 11P32, 11L20, 11N36, 11P05, 11P55.

(C)2005 American Mathematical Society 
the present paper is to obtain a similar result for seventh powers of primes. We establish the following theorem.

Theorem 1. Every sufficiently large even integer can be written as the sum of 46 seventh powers of prime numbers, that is, $H(7) \leq 46$.

We deduce Theorem 1 from the following result.

Theorem 2. Let $23 \leq s \leq 45$ and let $E_{s}(x)$ denote the number of integers $n \leq x$, with $n \equiv s(\bmod 2)$, that cannot be represented as sums of $s$ seventh powers of prime numbers. Then for any $A>0$, one has

$$
E_{23}(x) \ll x(\log x)^{-A},
$$

with an implied constant depending at most on $A$. When $s \geq 24$, there exists an absolute constant $\theta<1$ such that

$$
E_{s}(x) \ll x^{\theta-(s-23) / 672} .
$$

The main novelty in Theorem 2 is the estimate for $E_{23}(x)$, which is also the case used in the proof of Theorem 1. A variant of (1.4) was announced earlier as a part of Theorem 3 in Kumchev 8]. It is possible that a more delicate treatment of the major arcs (say, by a variant of the approach of Liu and Zhan [10]) would have allowed us to extend (1.4) to the case $s=23$. However, this would entail a significant amount of extra effort that would be spent wiser elsewhere.

As in earlier work on the subject, the proof of Theorem 2 uses the HardyLittlewood method. The new ingredients that allow us to bound $E_{23}(x)$ are the exponential sum estimates in Kumchev [9], the main result in Thanigasalam [13], and the sieve method in Harman 3 . The reader acquainted with Harman's method will recognize that we apply it in its most primitive form and may wonder whether it is not possible to dispense with the use of sieves altogether. That appears not to be the case. On the other hand, without the results in [9] and [13], even the most sophisticated version of the sieve does not seem to yield the desired result.

Notation. Throughout the paper, the letter $\varepsilon$ denotes a sufficiently small positive real number. Any statement in which $\varepsilon$ occurs holds for each positive $\varepsilon$, and any implied constant in such a statement is allowed to depend on $\varepsilon$. The letter $p$, with or without subscripts, is reserved for prime numbers; $c$ denotes an absolute constant, not necessarily the same in all occurrences. As usual in number theory, $\phi(n)$ and $\tau(n)$ denote Euler's totient function and the number of divisors function. Also, if $z \geq 2$, we define

$$
\psi(n, z)= \begin{cases}1, & \text { if } n \text { is divisible by no prime } p<z \\ 0, & \text { otherwise }\end{cases}
$$

and we write $e(x)=\exp (2 \pi \mathrm{i} x)$ and $(a, b)=\operatorname{gcd}(a, b)$.

We use several decompositions of the unit interval into sets of major and minor arcs. If $1 \leq Y \leq X$, we define the set of major $\operatorname{arcs} \mathfrak{M}(Y, X)$ as the union of the intervals

$$
\mathfrak{M}(q, a ; Y, X)=\left\{\alpha \in[0,1]:|q \alpha-a| \leq Y X^{-1}\right\}
$$

with $0 \leq a \leq q \leq Y$ and $(a, q)=1$. 


\section{Auxiliary Results}

2.1. Mean-value estimates. Our immediate goal is to describe a set of admissible exponents $\lambda_{1}, \ldots, \lambda_{22}$ satisfying (2.1) below. Our choice is motivated by the work of Thanigasalam [12, 13] and Vaughan [14]. We set $\theta_{22}=1, \theta_{21}=12 / 13, \alpha_{20}=37 / 91$, and then define recursively

$$
\nu_{i}=\frac{1+j_{i}-6 \alpha_{i}}{2^{j_{i}}-1+\alpha_{i}}, \quad \theta_{i}=\left(6+\nu_{i}\right) / 7, \quad \alpha_{i-1}=1 / 7+\theta_{i} \alpha_{i} \quad(13 \leq i \leq 20),
$$

with $j_{i}$ given by Table 1 .

TABLE 1 . The values of $j_{i}$

\begin{tabular}{|c|c|c|c|c|c|c|c|c|}
\hline$i$ & 13 & 14 & 15 & 16 & 17 & 18 & 19 & 20 \\
\hline$j_{i}$ & 5 & 5 & 5 & 5 & 4 & 4 & 3 & 3 \\
\hline
\end{tabular}

Further, we choose

$$
\theta_{i}=\left\{\begin{array}{ll}
1, & \text { if } i=1, \\
193 / 224, & \text { if } 2 \leq i \leq 12,
\end{array} \quad \text { and } \quad \lambda_{i}=\theta_{1} \cdots \theta_{i} \quad(1 \leq i \leq 22) .\right.
$$

In particular, a quick calculation reveals that

$$
\lambda_{1}+\cdots+\lambda_{22}>6.987443>7-2 / 159 .
$$

We now define the generating functions

$$
g(\alpha ; X)=\sum_{X<x \leq 2 X} e\left(\alpha x^{7}\right) \quad \text { and } \quad G_{i}(\alpha)=\prod_{j=i}^{22} g\left(\alpha ; P^{\lambda_{j}}\right) \quad(1 \leq i \leq 20) .
$$

The following lemma is the main result of this section.

Lemma 1. Let $P \geq 10$ and assume the above notation. Then

$$
\int_{0}^{1}\left|G_{i}(\alpha)\right|^{2} d \alpha \ll G_{i}(0) P^{\varepsilon} \quad(1 \leq i \leq 20) .
$$

Furthermore, if $u \geq 1$, we have

$$
\int_{0}^{1}|g(\alpha ; P)|^{u}\left|G_{1}(\alpha)\right|^{2} d \alpha \ll G_{1}(0)^{2} P^{u-7} .
$$

Proof. To prove (2.2), we refer to the theorem in Thanigasalam [13] for the case $i=20$ and then apply the iterative method in Vaughan [14 to deduce the remaining cases (see the discussion pertaining to $k=7$ on pp. $455-459$ in [14]).

We deduce (2.3) from (2.2) by a simple version of the circle method. We write

$$
\mathfrak{N}=\mathfrak{M}\left(Q, P^{7}\right), \quad \mathfrak{n}=[0,1] \backslash \mathfrak{N}, \quad \text { and } \quad \mathfrak{N}(q, a)=\mathfrak{M}\left(q, a ; Q, P^{7}\right),
$$

where $Q=P^{1 / 9}$. For $\alpha \in \mathfrak{n}$, Lemma 2.1 in Kawada and Wooley [7] yields

$$
g(\alpha ; P) \ll P^{63 / 64+\varepsilon} .
$$

Combining (2.1), (2.2), and (2.5), we obtain

$$
\int_{\mathfrak{n}}|g(\alpha ; P)|^{u}\left|G_{1}(\alpha)\right|^{2} d \alpha \ll G_{1}(0) P^{u-1 / 64+\varepsilon} \ll G_{1}(0)^{2} P^{u-7.003} .
$$


Suppose now that $\alpha \in \mathfrak{N}(q, a) \subset \mathfrak{N}$. By Lemmas 6.1 and 6.3 in Vaughan [15],

$$
g\left(\alpha ; P^{\lambda_{j}}\right) \ll \begin{cases}q^{-1 / 7} P\left(1+P^{7}|\alpha-a / q|\right)^{-1}, & \text { if } j=1, \\ q^{-1 / 7} P^{\lambda_{j}}, & \text { if } 2 \leq j \leq 10,\end{cases}
$$

whence

$$
|g(\alpha ; P)|^{u}\left|G_{1}(\alpha)\right|^{2} \ll G_{1}(0)^{2} P^{u}\left(q+P^{7}|q \alpha-a|\right)^{-3} .
$$

We deduce that

$$
\begin{aligned}
& \int_{\mathfrak{N}}|g(\alpha ; P)|^{u}\left|G_{1}(\alpha)\right|^{2} d \alpha \ll \sum_{q \leq Q} \sum_{\substack{1 \leq a \leq q \\
(a, q)=1}} \int_{\mathfrak{N}(q, a)} \frac{G_{1}(0)^{2} P^{u} d \alpha}{\left(q+P^{7}|q \alpha-a|\right)^{3}} \\
& \ll G_{1}(0)^{2} P^{u-7} .
\end{aligned}
$$

The desired bound follows from (2.4), (2.6), and (2.7).

\subsection{Exponential sum estimates.}

Lemma 2. Let $\alpha$ be real, let $\xi_{m}$ be complex numbers with $\left|\xi_{m}\right| \leq \tau(m)^{c}$, and define

$$
S(\alpha)=\sum_{\substack{M<m \leq 2 M \\ P<m n \leq 2 P}} \sum_{\substack{N<n \leq 2 N \\ \text { (n) }}} \xi_{m} \psi(n, z) e\left(\alpha(m n)^{7}\right),
$$

with $\psi(n, z)$ given by (1.5). Suppose that $M \leq P^{11 / 20}, z \leq \sqrt{2 P / M}$, and that there exist integers $a$ and $q$ satisfying

$$
1 \leq q \leq Q, \quad(a, q)=1, \quad|q \alpha-a|<Q P^{-7}
$$

with $Q \leq P$. Then

$$
S(\alpha) \ll q^{\varepsilon} L^{c}\left(P \Psi(\alpha)^{-1 / 2}+P^{11 / 20} \Psi(\alpha)^{1 / 2}+P z^{-1}\right),
$$

where $\Psi(\alpha)=q+P^{7}|q \alpha-a|$ and $L=\log P$. In particular, we have

$$
\sum_{P<p \leq 2 P} e\left(\alpha p^{7}\right) \ll q^{\varepsilon} L^{c}\left(P \Psi(\alpha)^{-1 / 2}+P^{11 / 20} \Psi(\alpha)^{1 / 2}\right) .
$$

Proof. (2.10) is the case $k=7$ of Lemma 5.6 in Kumchev [9]. (2.11) follows from (2.10) on choosing $M=\frac{1}{2}$ and $z=\sqrt{2 P}$.

Lemma 3. Let $1 / 192<\rho<1 / 141$ and let $\alpha$ be a real number such that no integers $a$ and $q$ satisfy (2.9) with $Q=P^{1 / 4}$. Let $S(\alpha)$ be defined by (2.8) and suppose that

$$
z \leq P^{1-128 \rho} \quad \text { and } \quad M \leq P^{(7-15 \rho) / 13} .
$$

Then

$$
S(\alpha) \ll P^{1-\rho+\varepsilon} .
$$

Proof. This follows from the results in $\S 3$ of $[9]$. Let $R=P^{(49-14 \rho) / 13}$. By Dirichlet's theorem on diophantine approximation, there exist integers $a$ and $q$ satisfying

$$
1 \leq q \leq R, \quad(a, q)=1, \quad|q \alpha-a|<R^{-1} .
$$

By assumption, $a$ and $q$ must also satisfy

$$
q+P^{7}|q \alpha-a|>P^{1 / 4} .
$$


When $z \leq P^{1-130 \rho}$, 2.13) and (2.14) suffice to deduce (2.12) from Lemma 3.3 in [9]. When $P^{1-130 \rho}<z \leq P^{1-128 \rho}$, we recall Buchstab's identity

$$
\psi\left(n, z_{2}\right)=\psi\left(n, z_{1}\right)-\sum_{z_{1} \leq p<z_{2}} \sum_{n=p k} \psi(k, p) \quad\left(2 \leq z_{1}<z_{2}\right) .
$$

Applying (2.15) with $z_{1}=P^{1-130 \rho}$ and $z_{2}=z$, we obtain

$$
S(\alpha)=S_{1}(\alpha)-S_{2}(\alpha)
$$

where

$$
\begin{gathered}
S_{1}(\alpha)=\sum_{\substack{M<m \leq 2 M \\
P<m n \leq 2 P}} \sum_{\substack{N<n \leq 2 N \\
P<n}} \xi_{m} \psi\left(n, z_{1}\right) e\left(\alpha(m n)^{7}\right), \\
S_{2}(\alpha)=\sum_{\substack{M<m \leq 2 M \\
z_{1} \leq p<z}} \sum_{\substack{z_{1} \leq k p \leq 2 N \\
P<m k p \leq 2 P}} \xi_{m} \psi(k, p) e\left(\alpha(m k p)^{7}\right) .
\end{gathered}
$$

We can now use Lemma 3.3 in [9] to bound $S_{1}(\alpha)$ and Lemma 3.1 in [9] (with $(m, n)=(m k, p))$ to bound $S_{2}(\alpha)$.

2.3. Estimates for sums over integers free of small prime divisors. In this section, we prepare some asymptotic estimates for exponential sums over numbers free of small primes.

Lemma 4. Let $2 \leq z \leq y \leq z^{c}$, let $\psi(n, z)$ be defined by (1.5), and let $\omega(u)$ be the continuous solution of the differential delay equation

$$
\begin{cases}(u \omega(u))^{\prime}=\omega(u-1), & \text { if } u>2, \\ \omega(u)=u^{-1}, & \text { if } 1<u \leq 2 .\end{cases}
$$

Let $B>0$ and let $\beta$ be a real number, with $|\beta| \leq y^{-7}(\log y)^{B}$. Then for any $A>0$,

$$
\sum_{n \leq y} \psi(n, z) e\left(\beta n^{7}\right)=e^{\gamma} W(z) \int_{z}^{y} e\left(\beta t^{7}\right) \omega\left(\frac{\log t}{\log z}\right) d t+O\left(y(\log y)^{-A}\right),
$$

the implied constant depending at most on $A$ and B. Here, $\gamma$ is Euler's constant and $W(z)=\prod_{p<z}\left(1-p^{-1}\right)$.

Proof. When $\beta=0$, this follows from (1.7) in de Bruijn [2] and de la Vallée Poussin's form of the prime number theorem (see $\S 18$ in Davenport 1]). The general case follows from the case $\beta=0$ by partial summation.

Lemma 5. Let $2 \leq z \leq y \leq z^{c}$, let $\psi(n, z)$ be defined by (1.5), and let $a$ and $q$ be integers, with $(a, q)=1$ and $1 \leq q \leq(\log y)^{B}$ for some $B>0$. Then for any $A>0$,

$$
\sum_{\substack{n \leq y \\ n \equiv a(\bmod q)}} \psi(n, z)=\frac{1}{\phi(q)} \sum_{n \leq y} \psi(n, z)+O\left(y(\log y)^{-A}\right),
$$

the implied constant depending at most on $A$ and $B$.

Proof. This is a generalization of the Siegel-Walfisz theorem. By a variant of (3) in $\S 20$ of Davenport [1], it suffices to show that

$$
\sum_{n \leq y} \psi(n, z) \chi(n) \ll y(\log y)^{-A},
$$


for every nonprincipal character $\chi \bmod q$. When $z \geq y^{1 / 2},(2.16)$ follows by partial summation from (3) in $\S 22$ of [1]. When $z<y^{1 / 2}$, we apply Buchstab's identity in the reverse. By (2.15),

$$
\sum_{n \leq y} \psi(n, z) \chi(n)=\sum_{n \leq y} \psi(n, \sqrt{y}) \chi(n)+\sum_{z \leq p<\sqrt{y}} \sum_{n \leq y p^{-1}} \psi(n, p) \chi(n p) .
$$

The first sum on the right of (2.17) can be estimated as before and the second sum is bounded above by

$$
y(\log y)^{2} \max _{M, M^{\prime}} M^{-1}\left|\sum_{M<p \leq M^{\prime}} \chi(p)\right|,
$$

the maximum being over all pairs $M, M^{\prime}$ with $z \leq M<M^{\prime} \leq \min (2 M, y)$. Since this quantity can also be estimated by means of $(3)$ in $\S 22$ of [1] , the desired result follows.

\section{Proof of Theorem 1}

Let $n$ be a large even integer and set

$$
P=\frac{1}{4} n^{1 / 7} \quad \text { and } \quad P_{j}=P^{\lambda_{j}} \quad(1 \leq j \leq 23),
$$

where $\lambda_{1}, \ldots, \lambda_{22}$ are the exponents defined in $\$ 2.1$ and $\lambda_{23}=1$. We aim to prove that the set

$$
\mathcal{U}=\left\{n-p_{1}^{7}-\cdots-p_{23}^{7}: P_{j}<p_{j} \leq 2 P_{j}\right\}
$$

contains an integer that can be represented as the sum of 23 seventh powers of primes. If $m$ is a natural number, we denote by $r(m)$ the number of solutions of

$$
p_{1}^{7}+p_{2}^{7}+\cdots+p_{23}^{7}=m
$$

subject to $P_{j}<p_{j} \leq 2 P_{j}$. By Cauchy's inequality,

$$
|\mathcal{U}| \geq\left(\sum_{m \in \mathcal{U}} r(n-m)\right)^{2}\left(\sum_{m<n} r(n-m)^{2}\right)^{-1} .
$$

The first sum on the right side of (3.2) is equal to the total number of 23-tuples $p_{1}, \ldots, p_{23}$ with $P_{j}<p_{j} \leq 2 P_{j}$, while the second sum is bounded above by the integral on the left side of (2.3) (with $u=2$ ). Hence, appeals to the prime number theorem and Lemma 11 yield

$$
|\mathcal{U}| \gg n(\log n)^{-46}
$$

Since the numbers in $\mathcal{U}$ are odd, the desired conclusion follows from (3.3) and (1.3).

\section{Proof of Theorem 2}

4.1. Preliminaries. For the sake of simplicity, we present the case $s=23$ in detail and then sketch the changes required in the proof of (1.4). Let $N$ be a large parameter. We set $P=\frac{1}{2} N^{1 / 7}$ and define $P_{1}, \ldots, P_{22}$ by (3.1). We also write

$$
z=P^{15 / 79}, \quad L=\log P, \quad \text { and } \quad X=P P_{1} \cdots P_{22} N^{-1} .
$$


Recalling (2.15), we obtain

$$
\begin{aligned}
\psi(m, \sqrt{2 P}) & =\psi(m, z)-\sum_{z \leq p<\sqrt{2 P}} \sum_{m=k p} \psi(k, p) \\
& \geq \psi(m, z)-\sum_{z \leq p<\sqrt{2 P}} \sum_{m=k p} \psi(k, z)=w(m), \quad \text { say. }
\end{aligned}
$$

When $P<m \leq 2 P$, the left side of (4.1) is equal to the indicator function of the primes, so the number of representations of $n$ as the sum of 23 seventh powers of prime numbers is bounded below by the quantity

$$
R(n)=\sum_{m, p_{1}, \ldots, p_{22}:(4.2)} w(m),
$$

where the summation is over the 23 -tuples $m, p_{1}, \ldots, p_{22}$ subject to

$$
\left\{\begin{array}{l}
m^{7}+p_{1}^{7}+\cdots+p_{22}^{7}=n \\
P<m \leq 2 P, \quad P_{j}<p_{j} \leq 2 P_{j} .
\end{array}\right.
$$

We now introduce some notation needed in the application of the circle method. We write

$$
\begin{gathered}
h(\alpha)=\sum_{P<m \leq 2 P} w(m) e\left(\alpha m^{7}\right), \quad f_{j}(\alpha)=\sum_{P_{j}<p \leq 2 P_{j}} e\left(\alpha p^{7}\right), \\
R(n ; \mathfrak{B})=\int_{\mathfrak{B}} h(\alpha) f_{1}(\alpha) \cdots f_{22}(\alpha) e(-\alpha n) d \alpha .
\end{gathered}
$$

Further, let

$$
\begin{gathered}
S^{*}(q, a)=\sum_{\substack{1 \leq b \leq q \\
(b, q)=1}} e\left(a b^{7} / q\right), \quad v_{j}(\beta)=\int_{P_{j}}^{2 P_{j}} \frac{e\left(\beta t^{7}\right)}{\log t} d t, \\
v^{*}(\beta ; z)=\int_{P}^{2 P} e\left(\beta t^{7}\right)\left[\omega\left(\frac{\log t}{\log z}\right)-\int_{z}^{\sqrt{2 P}} \omega\left(\frac{\log (t / u)}{\log z}\right) \frac{d u}{u \log u}\right] d t,
\end{gathered}
$$

where $\omega(u)$ is the function defined in Lemma 4 . We define the singular series $\mathfrak{S}(n)$ by

$$
\mathfrak{S}(n)=\sum_{q=1}^{\infty} B(n, q), \quad B(n, q)=\phi(q)^{-23} \sum_{\substack{1 \leq a \leq q \\(a, q)=1}} S^{*}(q, a)^{23} e(-a n / q) .
$$

The series $\mathfrak{S}(n)$ is thoroughly studied in Chapter 8 of Hua [5]. In particular, Theorem 12 in [5] asserts that

$$
1 \ll \mathfrak{S}(n) \ll 1
$$

for all odd $n$. The singular integral associated with $R(n)$ is $J(n)=J(n ; \infty)$, where

$$
J(n ; \xi)=\int_{-\xi}^{\xi} v_{1}(\beta)^{2} v_{2}(\beta) \cdots v_{22}(\beta) e(-\beta n) d \beta .
$$

Note that a routine application of the Fourier inversion formula yields

$$
X L^{-23} \ll J(n) \ll X L^{-23} .
$$


Because of the presence of the sieve weights $w(m)$, we also have to deal with a variant of $J(n ; \xi)$, namely,

$$
J^{*}(n ; \xi)=\int_{-\xi}^{\xi} v^{*}(\beta ; z) v_{1}(\beta) \cdots v_{22}(\beta) e(-\beta n) d \beta .
$$

Finally, we define the sets of major and minor arcs by $\mathfrak{M}=\mathfrak{M}\left(P^{1 / 4}, N\right)$ and $\mathfrak{m}=[0,1] \backslash \mathfrak{M}$, respectively.

4.2. The major arcs. Let $B$ be a real number with $B \geq A+c$, where $c$ is the constant appearing in (4.13) below. We may assume that $B \geq 50$. Our first order of business is to approximate $R\left(n ; \mathfrak{M}_{0}\right)$, where $\mathfrak{M}_{0}=\mathfrak{M}\left(L^{B}, N\right)$. When

$$
|q \alpha-a| \leq L^{B} N^{-1}, \quad 1 \leq q \leq L^{B}, \quad(a, q)=1,
$$

Lemma 5, the prime number theorem, and some partial summation yield

$$
f_{j}(\alpha)=\phi(q)^{-1} S^{*}(q, a) v_{j}(\alpha-a / q)+O\left(P_{j} L^{-3 B}\right) \quad(1 \leq j \leq 22)
$$

and

$$
h(\alpha)=\phi(q)^{-1} S^{*}(q, a) h(\alpha-a / q)+O\left(P L^{-3 B}\right) .
$$

Furthermore, by Lemma 4

$$
h(\alpha-a / q)=e^{\gamma} W(z) v^{*}(\alpha-a / q ; z)+O\left(P L^{-3 B}\right) .
$$

Since the measure of $\mathfrak{M}_{0}$ is $O\left(L^{2 B} N^{-1}\right)$, we deduce that

$$
R\left(n ; \mathfrak{M}_{0}\right)=e^{\gamma} W(z) \sum_{q \leq L^{B}} B(n, q) J^{*}\left(n ; L^{B} /(q N)\right)+O\left(X L^{-B}\right) .
$$

In the next section, we show that if $q \leq L^{B / 2}$, then

$$
e^{\gamma} W(z) J^{*}\left(n ; L^{B} /(q N)\right)=\delta J(n)+O\left(X L^{-23.5}\right),
$$

where $\delta$ is a positive absolute constant. Since by Lemma 8.5 in Hua 5 , we have $B(n, q) \ll q^{-10}$, (4.4)-(4.6) and the trivial bound for $J^{*}(n ; \xi)$ imply that

$$
R\left(n ; \mathfrak{M}_{0}\right)=\delta \mathfrak{S}(n) J(n)+O\left(X L^{-23.5}\right) .
$$

We deal with the contribution from the remainder of the major arcs by means of Lemma 2 Let $\mathfrak{K}=\mathfrak{M} \backslash \mathfrak{M}_{0}$ and write

$$
\mathfrak{K}(q, a)= \begin{cases}\mathfrak{M}\left(q, a ; P^{1 / 4}, N\right) \backslash \mathfrak{M}\left(q, a ; L^{B}, N\right), & \text { if } q \leq L^{B}, \\ \mathfrak{M}\left(q, a ; P^{1 / 4}, N\right), & \text { if } L^{B}<q \leq P^{1 / 4} .\end{cases}
$$

We want to bound the cardinality of the set

$$
\mathcal{X}=\left\{n \in(N, 2 N]:|R(n ; \mathfrak{K})| \geq X L^{-25}\right\} .
$$

By Bessel's inequality,

$$
\sum_{n \in \mathcal{X}}|R(n ; \mathfrak{K})|^{2} \leq \int_{\mathfrak{K}}\left|h(\alpha) f_{1}(\alpha) \ldots f_{22}(\alpha)\right|^{2} d \alpha .
$$

For $\alpha \in \mathfrak{K}(q, a)$, 2.11) gives

$$
f_{j}(\alpha) \ll \begin{cases}q^{-1 / 2+\varepsilon} L^{c} P_{1}(1+N|\alpha-a / q|)^{-1 / 2}, & \text { when } j=1, \\ q^{-1 / 2+\varepsilon} L^{c} P_{j}, & \text { when } j=2,3,4 .\end{cases}
$$


Furthermore, by (4.1), $h(\alpha)$ is the difference of two sums of the form (2.8) with $M \leq P^{1 / 2}$ and $z=P^{15 / 79}$. Applying (2.10) to each of those sums, we find that when $\alpha \in \mathfrak{K}(q, a)$,

$$
h(\alpha) \ll q^{-1 / 2+\varepsilon} L^{c} P(1+N|\alpha-a / q|)^{-1 / 2} .
$$

Combining (4.9) -(4.11) and the trivial bounds for $f_{5}(\alpha), \ldots, f_{22}(\alpha)$, we get

$$
\begin{aligned}
\sum_{n \in \mathcal{X}}|R(n ; \mathfrak{K})|^{2} & \ll \sum_{q \leq P^{1 / 4}} \sum_{\substack{1 \leq a \leq q \\
(a, q)=1}} \int_{\mathfrak{K}(q, a)} \frac{q^{-4}(X N)^{2} L^{c}}{(1+N|\alpha-a / q|)^{2}} d \alpha \\
& \ll(X N)^{2} L^{c} \sum_{q \leq P^{1 / 4}} \int_{\mathfrak{K}(q, 0)} \frac{q^{-3} d \beta}{(1+N|\beta|)^{2}} \ll X^{2} N L^{c-B} .
\end{aligned}
$$

Recalling (4.8), we deduce that

$$
|\mathcal{X}| \ll N L^{c-B} \ll N L^{-A} .
$$

4.3. The singular integral. In this section, we establish (4.66). By Lemma 6.2 in Vaughan [15],

$$
v_{j}(\beta) \ll P_{j} L^{-1}\left(1+P_{j}^{7}|\beta|\right)^{-1} \quad(1 \leq j \leq 22),
$$

and a similar argument yields

$$
v^{*}(\beta ; z) \ll P(1+N|\beta|)^{-1}
$$

Also, some elementary analysis reveals that

$$
v^{*}(\beta ; z)=\delta_{0} L v_{1}(\beta)+O\left(P L^{-1}\right),
$$

where

$$
\delta_{0}=\omega\left(\frac{79}{15}\right)-\int_{1 / 2}^{64 / 79} \omega\left(\frac{79 u}{15}\right) \frac{d u}{1-u} .
$$

We remark that numerical integration shows that $\delta_{0}>0.01$. Using the above estimates, we find that if $q \leq L^{B / 2}$, then

$$
\begin{aligned}
J^{*}\left(n ; L^{B} /(q N)\right) & =J^{*}\left(n ; L^{1 / 2} N^{-1}\right)+O\left(X L^{-22.5}\right) \\
& =\delta_{0} L J\left(n ; L^{1 / 2} N^{-1}\right)+O\left(X L^{-22.5}\right) \\
& =\delta_{0} L J(n)+O\left(X L^{-22.5}\right) .
\end{aligned}
$$

Thus, (4.6) with $\delta=\frac{79}{15} \delta_{0}$ follows from the formula (see Ingham [6], p. 24)

$$
W(z)=e^{-\gamma}(\log z)^{-1}+O\left((\log z)^{-2}\right) .
$$

4.4. The minor arcs. Next we proceed to bound the cardinality of the set

$$
\mathcal{Y}=\left\{n \in(N, 2 N]:|R(n ; \mathfrak{m})| \geq X L^{-25}\right\}
$$

By Bessel's inequality,

$$
\sum_{n \in \mathcal{Y}}|R(n ; \mathfrak{m})|^{2} \leq \int_{\mathfrak{m}}\left|h(\alpha) f_{1}(\alpha) \cdots f_{22}(\alpha)\right|^{2} d \alpha
$$

We can estimate the last integral by means of Lemmas 1 and 3. By Lemma B with $\rho=1 / 158$ (note that $15 / 79=1-128 / 158)$,

$$
\sup _{\alpha \in \mathfrak{m}}|h(\alpha)| \ll P^{157 / 158+\varepsilon} .
$$


Also, by comparing the underlying diophantine equations, we get

$$
\int_{0}^{1}\left|f_{1}(\alpha) \cdots f_{22}(\alpha)\right|^{2} d \alpha \leq \int_{0}^{1}\left|G_{1}(\alpha)\right|^{2} d \alpha,
$$

where $G_{1}(\alpha)$ is the generating function appearing in Lemma 1 Thus, (2.2) yields

$$
\int_{0}^{1}\left|f_{1}(\alpha) \cdots f_{22}(\alpha)\right|^{2} d \alpha \ll P^{\lambda_{1}+\cdots+\lambda_{22}+\varepsilon} .
$$

Recalling (2.1), we conclude from (4.14)-(4.17) that

$$
|\mathcal{Y}| \ll N P^{-\eta},
$$

where $\eta=1 / 79-2 / 159$.

Finally, suppose that $n \in(N, 2 N]$ is an odd integer such that $n \notin \mathcal{X} \cup \mathcal{Y}$. Then, by (4.3), (4.4), (4.7), (4.8), and (4.14), we obtain

$$
R(n) \gg X L^{-23} \text {. }
$$

In view of (4.13) and (4.18), this implies that all but $O\left(N L^{-A}\right)$ odd $n \in(N, 2 N]$ are representable as the sum of 23 seventh powers of primes. This establishes the case $s=23$ of the theorem.

4.5. The case $s \geq 24$. The generating function $h(\alpha) f_{1}(\alpha) \cdots f_{22}(\alpha)$ that we used above is now replaced by

$$
h(\alpha) f_{1}(\alpha)^{s-22} f_{2}(\alpha) \cdots f_{22}(\alpha) .
$$

First, we note that when $s \geq 24$, we can use (4.10) and (4.11) to directly estimate $R(n ; \mathfrak{K})$ in a similar fashion to 4.12). Hence, when $s \geq 24$, we have $\mathcal{X}=\varnothing$. Further, by the case $k=7$ of Theorem 3 in $[9$,

$$
\sup _{\alpha \in \mathfrak{m}}\left|f_{1}(\alpha)\right| \ll P^{191 / 192+\varepsilon} .
$$

Thus, for every additional copy of $f_{1}(\alpha)$ in the generating function (4.19), we can save a factor of $P^{1 / 96}$ on (4.18). Making use of these observations, we obtain (1.4) with $\theta=1-\eta / 7 \approx 0.999989$.

\section{REFERENCES}

[1] H. Davenport, Multiplicative Number Theory, third ed., Graduate Texts in Mathematics, vol. 74, Springer-Verlag, New York, 2000, revised by H. L. Montgomery. MR1790423 (2001f:11001)

[2] N. J. de Bruijn, On the number of uncancelled elements in the sieve of Eratosthenes, Proc. Kon. Ned. Akad. Wetensch. 53 (1950), 803-812. MR0035785 (12:11d)

[3] G. Harman, On the distribution of $\alpha p$ modulo one II, Proc. London Math. Soc. (3) 72 (1996), 241-260. MR1367078 (96k:11089)

[4] L. K. Hua, Some results in prime number theory, Quart. J. Math. Oxford Ser. 9 (1938), 68-80.

[5] - Additive Theory of Prime Numbers, American Mathematical Society, Providence, RI, 1965. MR0194404 (33:2614)

[6] A. E. Ingham, The Distribution of Primes, reprint of the 1932 original ed., Cambridge University Press, Cambridge, 1990, with a foreword by R. C. Vaughan. MR1074573 (91f:11064)

[7] K. Kawada and T. D. Wooley, On the Waring-Goldbach problem for fourth and fifth powers, Proc. London Math. Soc. (3) 83 (2001), 1-50. MR.1829558 (2002b:11134)

[8] A. Kumchev, On the Waring-Goldbach problem. Exceptional sets for sums of cubes and higher powers, to appear in Canad. J. Math.

[9] _ On Weyl sums over primes and almost primes, preprint. 
[10] J. Y. Liu and T. Zhan, The exceptional set in Hua's theorem for three squares of primes, to appear in Acta. Math. Sinica.

[11] K. Thanigasalam, Improvement on Davenport's iterative method and new results in additive number theory I, Acta Arith. 46 (1985), 1-31. MR0831261|(87e:11118)

[12] _ Improvement on Davenport's iterative method and new results in additive number theory III, Acta Arith. 48 (1987), 97-116. MF0895435 (88f:11097)

[13] _ On admissible exponents for kth powers, Bull. Calcutta Math. Soc. 86 (1994), 175178. MR 1323498 (96c:11117)

[14] R. C. Vaughan, On Waring's problem for smaller exponents, Proc. London Math. Soc. (3) 52 (1986), 445-463. MR0833645 (87g:11126)

[15] — The Hardy-Littlewood Method, second ed., Cambridge Tracts Math., vol. 125, Cambridge University Press, Cambridge, 1997. MR.1435742 (98a:11133)

[16] I. M. Vinogradov, Representation of an odd number as the sum of three primes, Dokl. Akad. Nauk SSSR 15 (1937), 291-294, in Russian.

Department of Mathematics, 1 University Station, C1200, The University of Texas at Austin, Austin, Texas 78712

E-mail address: kumchev@math.utexas.edu 